\title{
Comparing Metamodeling Techniques For Variability Analysis In Sheet Metal Forming Processes
}

\author{
P.A. Prates ${ }^{1, a)}$; A.E. Marques ${ }^{1, \text { b) }}$; M.C. Oliveira ${ }^{1, c)}$ and J.V. Fernandes ${ }^{1, d)}$ \\ ${ }^{1}$ CEMMPRE, Department of Mechanical Engineering, University of Coimbra, Pólo II, Rua Luís Reis Santos, Pinhal \\ de Marrocos, 3030-788 Coimbra, Portugal

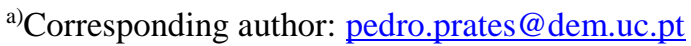 \\ b) ar.eusebio@ hotmail.com \\ c)marta.oliveira@dem.uc.pt \\ d) valdemar.fernandes@dem.uc.pt
}

\begin{abstract}
This study presents a systematic comparison on the performance of different metamodeling techniques in the analysis of variability in sheet metal forming processes. For this purpose, three steel grades (DC06, DP600 and HSLA340) are selected as reference materials and two sheet metal forming processes are considered: the U-Channel and the Square Cup forming processes. The sources of variability selected for this study are the Young's modulus, the isotropic hardening law parameters, the anisotropy coefficients and the initial thickness of the sheet metal; the variability is described for all of them by a probabilistic normal distribution. The process outputs selected for analysis are the springback and maximum thinning, in case of the U-Channel forming process, and the maximum equivalent plastic strain and maximum thinning, in case of the Square Cup deep-drawing. Firstly, a number of random simulations is performed for each material and forming process. Then, metamodeling techniques based on $2^{\text {nd }}$ degree polynomial RSM and three Kriging methods (Simple, Ordinary and Universal Kriging) are established, and their performance is evaluated. The results show that the performance of Kriging metamodels is generally better than RSM; also, the performance of RSM metamodels is strongly dependent on the number of design (training) points, which is not the case for Kriging metamodels.
\end{abstract}

\section{INTRODUCTION}

The analysis of sheet metal forming processes is often based on deterministic approaches, excluding the variability inherent to the material and process parameters. Variability analyses of sheet metal forming processes have recently been carried out in the literature, typically combining Finite Element Analysis (FEA) with metamodeling techniques such as Response Surface Methodology (RSM) and Kriging [1-3]. In this regard, metamodeling techniques establish mathematical relationships between the numerical inputs (e.g. material properties, geometrical characteristics and process parameters) and the numerical simulation outputs of forming processes (or responses). To our knowledge, no systematic comparison has been made so far on the performance of different metamodeling techniques in the analysis of variability of forming processes; in literature, the choice of the metamodeling technique is made presumptively, without prior analysis of its suitability to the problem under study. In this work, the performances of Kriging and RSM metamodels are evaluated for two distinct types of sheet metal forming processes: the U-Channel and the Square Cup deep-drawing, to try to understand the impact of the type of response. Moreover, the study is performed considering materials with distinct hardening and anisotropic behaviour. 


\section{METAMODELING}

RSM and Kriging are metamodeling techniques since they allow establishing mathematical relationships between the design variables (i.e. sources of variability) and the simulated outputs (i.e. responses) of forming processes. The vector of design variables is defined as $\mathbf{x}=x_{i}, i=1, \ldots, p$, where $p$ is the total number of sources of variability (inputs). In order to fit the response measurements, it is necessary to evaluate the response $\mathbf{z}(\mathbf{x})$ for a predefined set of design points, $\mathbf{x}^{\mathrm{d}}$, such that $\mathbf{z}\left(\mathbf{x}^{\mathrm{d}}\right)=z^{\mathrm{d}}{ }_{k}, k=1, \ldots, n$, where $n$ is the total number of random simulations performed. This also allows defining a design matrix $\mathbf{X}^{\mathrm{d}}=x^{\mathrm{d}}{ }_{i k}$, with $i=1, \ldots, p$ and $k=1, \ldots, n$.

RSM is a regression model that fits a polynomial function to a set of known points. In this work, a quadratic function is used, as follows:

$$
z(\mathbf{x})=\beta_{0}+\sum_{i=1}^{p} \beta_{i} x_{i}+\sum_{i=1}^{p} \sum_{j>i}^{p} \beta_{i j} x_{i} x_{j}+\sum_{i=1}^{p} \beta_{i i} x_{i}^{2},
$$

where $z(\mathbf{x})$ is the estimated response for a given set of inputs $\mathbf{x}$ and $\beta_{0}, \beta_{i}, \beta_{i j}$ and $\beta_{i i}$ are the set of RSM coefficients, which can be organized in the vector of unknowns $\boldsymbol{\beta}$, with a dimension equal to the total number of RSM coefficients: $\beta=0.5 p^{2}+1.5 p+1$. Note that for $n<\beta$ the system of equations is underdetermined while for $n>\beta$ it is overdetermined, i.e. there is a unique solution only when $n=\beta$. Thus, for $n \neq \beta$, the least square solution is determined. This means that for $n<\beta$ the Euclidean norm $\|\boldsymbol{\beta}\|$ is minimized, imposing that $\mathbf{z}=\mathbf{H} \boldsymbol{\beta}$; where $\mathbf{H}$ is the linear system matrix. For $n>\beta$ it is the Euclidean norm $\|\mathbf{z}-\mathbf{H} \boldsymbol{\beta}\|$ that is minimized.

Kriging is an interpolation technique that estimates the response $z(\mathbf{x})$, for a given set of inputs $\mathbf{x}$, as a sum of two functions, a trend function $f(\mathbf{x})$ and a residual function $R(\mathbf{x})$ :

$$
z(\mathbf{x})=f(\mathbf{x})+R(\mathbf{x}) .
$$

Kriging estimates the residual function $R(\mathbf{x})$ as a weighted sum of the residuals $R\left(\mathbf{x}^{\alpha}\right)$, evaluated at the design points located in the neighbourhood of the estimation point, $\mathbf{x}^{\mathrm{e}}$. Assuming that all design points are in the neighbourhood of the estimation point, $\mathbf{x}^{\mathrm{e}}$ :

$$
R(\mathbf{x})=\sum_{\alpha=1}^{n} w_{\alpha} R\left(\mathbf{x}^{\alpha}\right), \text { with } R\left(\mathbf{x}^{\alpha}\right)=z\left(\mathbf{x}^{\alpha}\right)-f\left(\mathbf{x}^{\alpha}\right),
$$

where $w_{\alpha}$ are the Kriging weights, $n$ is the total number of known points, $z\left(\mathbf{x}^{\alpha}\right)$ is the response for the predefined set of design points $\mathbf{x}^{\alpha}$ at known point $\alpha$, and $f\left(\mathbf{x}^{\alpha}\right)$ is the trend function at $\mathbf{x}^{\alpha}$. The Kriging weights are determined by solving a system of linear equations, which is established based on the matrix of covariance between each pair of known points, $\mathbf{C}$. The covariance function $C\left(\left|\mathbf{x}^{i}-\mathbf{x}^{j}\right|\right)$ chosen in this study corresponds to the Gaussian model:

$$
C\left(\left|\mathbf{x}^{i}-\mathbf{x}^{j}\right|\right)=b \exp \left(-\frac{\left|\mathbf{x}^{i}-\mathbf{x}^{j}\right|^{2}}{a^{2}}\right),
$$

where $\left|\mathbf{x}^{i}-\mathbf{x}^{j}\right|$ is the Euclidean distance between the points $\mathbf{x}^{i}$ and $\mathbf{x}^{j}$, and $a$ and $b$ are the parameters of the Gaussian model. The parameters $a$ and $b$ are fitted to quantify how the responses are related through distances between pairs of points. The three Kriging variants selected in this work, Simple Kriging (SK), Ordinary Kriging (OK) and Universal Kriging (UK), differ in their treatments of the trend function $f(\mathbf{x})$. For SK, it is assumed that the trend function is a constant and known value $f(\mathbf{x})=m$, which corresponds to the mean value of the known responses. Therefore, the response estimation for SK is given by:

$$
z(\mathbf{x})=m+\sum_{\alpha=1}^{n} w_{\alpha}\left[z\left(\mathbf{x}^{\alpha}\right)-m\right] .
$$

Therefore, the system of linear equations is defined as follows:

$$
C_{i k} w_{k}=c_{i} \text {, with } i, k=1, \ldots, n,
$$

where $\mathbf{C}$ is the square matrix of covariance between each pair of known points and $\boldsymbol{c}$ is the vector of covariance between the design points, $\mathbf{x}^{\alpha}$, and the estimation point, $\mathbf{x}^{\mathrm{e}}$. For OK, it is assumed that the trend function is a constant and unknown mean in the neighbourhood of each estimation point, $f(\mathbf{x})=m(\mathbf{x})$. The response estimation for OK is given by:

$$
z(\mathbf{x})=m(\mathbf{x})+\sum_{\alpha=1}^{n} w_{\alpha}\left[z\left(\mathbf{x}^{\alpha}\right)-m(\mathbf{x})\right]=\sum_{\alpha=1}^{n} w_{\alpha} z\left(\mathbf{x}^{\alpha}\right)+m(\mathbf{x})\left[1-\sum_{\alpha=1}^{n} w_{\alpha}\right] .
$$

The unknown mean $m(\mathbf{x})$ is filtered by imposing $\sum_{\alpha=1}^{n} w_{\alpha}=1$, to ensure an unbiased solution, i.e. $z(\mathbf{x})=\sum_{\alpha=1}^{n} w_{\alpha} z\left(\mathbf{x}^{\alpha}\right)$. In this case, the calculation of the Kriging weights requires including an additional equation in the system shown in Eq. (6), as follows: 


$$
\left[\begin{array}{ccc:c}
C_{11} & \cdots & C_{1 n} & 1 \\
\vdots & \ddots & \vdots & \vdots \\
C_{n 1} & \cdots & C_{n n} & 1 \\
\hdashline 1 & \cdots & 1 & 0
\end{array}\right]\left\{\begin{array}{c}
w_{1} \\
\vdots \\
w_{n} \\
\lambda
\end{array}\right\}=\left\{\begin{array}{c}
c_{1} \\
\vdots \\
c_{n} \\
\hdashline 1
\end{array}\right\},
$$

where $\lambda$ is a Lagrange multiplier. For UK, it is assumed that the trend function is a linear or higher-order function. In this work a linear trend function is used, $f(\mathbf{x})=a_{0}+\sum_{i=1}^{p} a_{i} x_{i}$, where $a_{i}, i=0, \ldots, p$, are the coefficients of the linear function. In this case, the calculation of the Kriging weights requires adding $p$ extra lines and columns to the ordinary Kriging system of equations (see Eq. (8)), as follows:

$$
\left[\begin{array}{cccc:ccc}
C_{11} & \cdots & C_{1 n} & 1 & x_{11}^{\mathrm{d}} & \cdots & x_{1 p}^{\mathrm{d}} \\
\vdots & \ddots & \vdots & \vdots & \vdots & \ddots & \vdots \\
C_{n 1} & \cdots & C_{n n} & 1 & x_{n 1}^{\mathrm{d}} & \cdots & x_{n p}^{\mathrm{d}} \\
1 & \cdots & 1 & 0 & 0 & \cdots & 0 \\
\hdashline x_{11}^{\mathrm{d}} & \cdots & x_{1 n}^{\mathrm{d}} & 0 & 0 & \cdots & 0 \\
\vdots & \ddots & \vdots & \vdots & \vdots & \ddots & \vdots \\
x_{p 1}^{\mathrm{d}} & \cdots & x_{p n}^{\mathrm{d}} & 0 & 0 & \cdots & 0
\end{array}\right]\left\{\begin{array}{c}
w_{1} \\
\vdots \\
w_{n} \\
\lambda_{0} \\
\hdashline \lambda_{1} \\
\vdots \\
\lambda_{p}
\end{array}\right\}=\left\{\begin{array}{c}
c_{1} \\
\vdots \\
c_{m} \\
\lambda \\
\hdashline x_{1}^{\mathrm{e}} \\
\vdots \\
x_{p}^{\mathrm{e}}
\end{array}\right\},
$$

where $x_{k i}^{\mathrm{d}}$, with $k=1, \ldots, n$ and $i=1, \ldots, p$ corresponds to the coefficients of the design matrix, $\mathbf{X}^{\mathrm{d}} ; x_{i}^{\mathrm{e}}$ are the values of each design variable at the estimation point and $\lambda_{i}$ are the Lagrange multipliers.

\section{STUDY CASES}

This section presents the details of the numerical models of the U-Channel and Square Cup forming processes, including the materials considered and the relevant input variables. The procedure adopted for generating and evaluating both Kriging and RSM metamodels is also described.

\section{Numerical Models}

The numerical models of the U-Channel and Square Cup forming processes are represented in Figure 1. Both processes comprise three main elements: the blank holder, the die and the punch. The blank holder force is assumed constant and equal to $4.9 \mathrm{kN}$ during both forming processes, which end after a total punch displacement of $30 \mathrm{~mm}$, in case of the U-Channel forming, and $40 \mathrm{~mm}$, in case of the Square Cup forming. The initial dimensions of the blank of the U-Channel and Square Cup forming processes are, respectively, $150 \times 35 \times 0.78 \mathrm{~mm}^{3}$ and $75 \times 75 \times 0.78 \mathrm{~mm}^{3}$. The material is considered orthotropic. Due to material and geometry symmetries, only one fourth of the blank is simulated for the Square Cup deep-drawing process, considering a finite element mesh with 1800, 8-node hexahedral solid elements. For the U-Channel, only half of the blank is considered and boundary conditions are set to guarantee a plain strain state along the width of the blank, which enables the use of a total of 450, 8-node hexahedral solid elements.

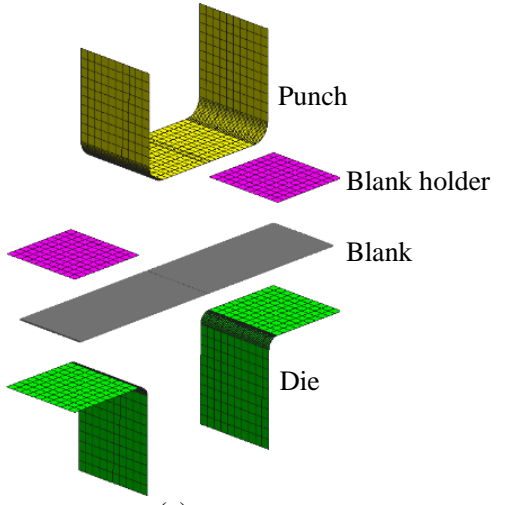

(a)

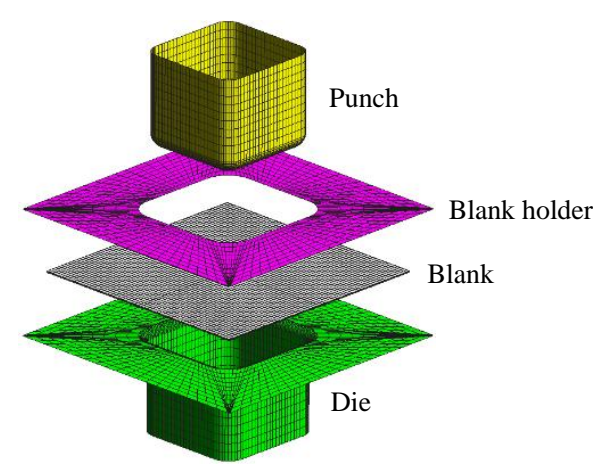

(b)

FIGURE 1. Representation of the finite element models for the study cases: (a) U-Channel; (b) Square Cup 
The numerical simulations were carried out with the in-house finite element code DD3IMP, developed and optimized for simulating sheet metal forming processes [4]. The forming tool geometry was modelled using Nagata patches [5]. The contact with friction is described by Coulomb's law, with a friction coefficient of 0.144 . The constitutive model adopted in this study assumes (i) the isotropic elastic behaviour described by the generalised Hooke's law and (ii) the plastic behaviour described by the orthotropic Hill'48 yield criterion combined with Swift isotropic hardening law. The Hill'48 yield criterion is described as follows:

$$
F\left(\sigma_{\mathrm{yy}}-\sigma_{\mathrm{zz}}\right)^{2}+G\left(\sigma_{\mathrm{zz}}-\sigma_{\mathrm{xx}}\right)^{2}+H\left(\sigma_{\mathrm{xx}}-\sigma_{\mathrm{yy}}\right)^{2}+2 L \tau_{\mathrm{yz}}^{2}+2 M \tau_{\mathrm{xz}}^{2}+2 N \tau_{\mathrm{xy}}^{2}=Y^{2} .
$$

where $\sigma_{\mathrm{xx}}, \sigma_{\mathrm{yy}}, \sigma_{\mathrm{zz}}, \tau_{\mathrm{xy}}, \tau_{\mathrm{yz}}$ and $\tau_{\mathrm{xz}}$ are the components of the Cauchy stress tensor defined in orthotropic coordinate system of the material; $F, G, H, L, M$ and $N$ are the anisotropy parameters and $Y$ is the flow stress. The condition $G+$ $H=1$ is assumed and so $Y$ is represented by the uniaxial tensile stress along the rolling direction of the sheet. The parameters $L$ and $M$ are assumed equal to 1.5, as in isotropy (von Mises). The parameters $F, G, H$ and $N$ can be related with the anisotropy coefficients $r_{0}, r_{45}$ and $r_{90}$, as follows:

$$
F=\frac{r_{0}}{r_{90}\left(r_{0}+1\right)}, G=\frac{1}{r_{0}+1}, H=\frac{r_{0}}{r_{0}+1}, N=\frac{1}{2} \frac{\left(r_{0}+r_{90}\right)\left(2 r_{45}+1\right)}{r_{90}\left(r_{0}+1\right)} .
$$

The Swift hardening law is expressed by:

$$
Y=C\left[\left(Y_{0} / C\right)^{\left(1 / n_{h}\right)}+\bar{\varepsilon}^{\mathrm{p}}\right]^{n_{h}}
$$

where $\bar{\varepsilon}^{\mathrm{p}}$ is the equivalent plastic strain and $C, Y_{0}$ and $n_{h}$ are material parameters. Two types of numerical simulation outputs were considered for each forming process: (i) springback and maximum thinning, for the U-Channel process, and (ii) maximum equivalent plastic strain and maximum thinning, for the Square Cup deep-drawing.

\section{Materials}

Three different steels are considered for each forming process: DC06, DP600 and HSLA340. For each of these materials, a normal distribution is assumed for describing the variability of the following inputs: $C, Y_{0}$ and $n_{h}$ of the Swift hardening law; Young's Modulus, $E$; anisotropy coefficients $r_{0}, r_{45}$ and $r_{90}$, and initial sheet thickness, $t_{0}$. The

\begin{tabular}{|c|c|c|c|c|c|c|c|c|c|}
\hline Materials & & $\begin{array}{c}C \\
{[\mathrm{MPa}]}\end{array}$ & $n_{h}$ & $\begin{array}{c}Y_{0} \\
{[\mathrm{MPa}]}\end{array}$ & $E[\mathbf{G P a}]$ & $r_{0}$ & $r 45$ & $r_{90}$ & $\begin{array}{c}t_{0} \\
{[\mathrm{~mm}]}\end{array}$ \\
\hline \multirow{2}{*}{ DC06 } & $\mu$ & 565.32 & 0.259 & 157.12 & 206 & 1.790 & 1.510 & 2.270 & 0.780 \\
\hline & $\sigma$ & 26.85 & 0.018 & 7.16 & 3.85 & 0.051 & 0.037 & 0.121 & 0.013 \\
\hline \multirow{2}{*}{ DP600 } & $\mu$ & 1093.0 & 0.187 & 330.30 & 210 & 1.010 & 0.760 & 0.980 & 0.780 \\
\hline & $\sigma$ & 52.46 & 0.02 & 9.64 & 7.35 & 0.04 & 0.03 & 0.06 & 0.01 \\
\hline \multirow{2}{*}{ HSLA340 } & $\mu$ & 673.0 & 0.131 & 365.30 & 210 & 0.820 & 1.070 & 1.040 & 0.780 \\
\hline & $\sigma$ & 32.30 & 0.011 & 10.67 & 7.35 & 0.033 & 0.039 & 0.061 & 0.005 \\
\hline
\end{tabular}
mean $(\mu)$ and standard deviation $(\sigma)$ values of each parameter are detailed in Table 1.

TABLE 1. Values of $\mu$ and $\sigma$ for each input of the three materials considered

\section{Metamodel Generation and Evaluation}

Based on the normal distribution of each input shown in Table 1, 60 sets of inputs were randomly generated for each material. Numerical simulations of the U-Channel and Square Cup forming processes were performed for each of these randomly generated inputs, $\mathbf{x}$, with a total of 3 (materials) $\times 60$ (sets of inputs) $=180$ simulations for each forming process. For each material, the numerical simulations of each forming process were grouped into two sets: one training set, $\mathbf{x}^{\mathrm{d}}$, with $n$ simulations for generating Kriging and RSM metamodels, and one testing set, $\mathbf{x}^{\mathrm{e}}$, with $k$ simulations for evaluating the performance of the generated metamodels, by comparing the estimated/predicted output values with those obtained by numerical simulation. The root mean square relative error (RMSRE) is used to evaluate the performance of each metamodel:

$$
\text { RMSRE }=\sqrt{\frac{1}{k} \sum_{i=1}^{k}\left(\frac{z_{i}-\tilde{z}_{i}}{z_{i}}\right)^{2}},
$$

where $z_{i}$ and $\tilde{z}_{i}$ are the simulated and predicted response values for the set of testing inputs $\mathbf{x}^{\mathrm{e}}$, respectively. In a first approach, the two sets were defined with an equal dimension, i.e. $n=k=30$. It should be noted that in this case the 
number of training simulations for each RSM metamodel is less than the number of RSM coefficients $\beta=45$, (for $p=8$ inputs - see Equation 1). In a second approach, the two sets were defined as $n=45$ and $k=15$; in this case, the number of training simulations for each RSM metamodel equals $\beta$, and the solution for $\boldsymbol{\beta}$ is unique. A total of 48 metamodels were generated for each forming process, each corresponding to a material (3), type of response (2), metamodeling technique (4) and training/testing set (2).

\section{RESULTS}

Table 2 presents the RMSRE values obtained for the metamodels generated with the U-Channel and Square Cup processes. Overall, the Kriging techniques (UK in particular) present better predictions than RSM, achieving values of RMSRE below 5\%, with the only exception being the prediction of the maximum thinning in the Square Cup deepdrawing using DP600 and 30 training simulations. In some cases, such as the prediction of maximum equivalent plastic strain for the Square Cup deep-drawing using HSLA340, all Kriging techniques give similar performances, while in other cases UK performs better than SK and OK (the only exception is the prediction of the maximum thinning in the Square Cup deep-drawing using DP600 and 30 training simulations). It can be concluded that, for the cases where all Kriging techniques give similar performances, the trend function $f(\mathbf{x})$ is constant, which makes both SK and OK techniques competitive with UK. For the remaining cases, UK performs better than SK and OK because the trend function $f(\mathbf{x})$ is not constant. Also, the values of RMSRE for Kriging metamodels generated from 30 and 45 training simulations are relatively close to each other. However, some of the models with $n=45$ underperform the models with $n=30$, which can be related with stronger oscillations of the metamodel when a higher number of interpolation (training) points is used, leading to an increase in the values of RMSRE.

TABLE 2. Values of RMSRE (\%) obtained for the Kriging and RSM metamodels of the U-Channel and Square Cup processes.

\begin{tabular}{|c|c|c|c|c|c|c|c|}
\hline \multirow[t]{2}{*}{$\begin{array}{l}\text { U-Channel } \\
\end{array}$} & \multirow[b]{2}{*}{ Train/Test } & \multicolumn{3}{|c|}{ Springback } & \multicolumn{3}{|c|}{ "Maximum Thinning } \\
\hline & & DC06 & DP600 & HSLA340 & DC06 & DP600 & HSLA340 \\
\hline \multirow{2}{*}{ SK } & $30 / 30$ & 2.83 & 3.47 & 1.52 & 0.73 & 1.43 & 3.14 \\
\hline & $45 / 15$ & 3.90 & 2.73 & 3.87 & 1.54 & 1.16 & 2.71 \\
\hline \multirow{2}{*}{ OK } & $30 / 30$ & 2.77 & 3.48 & 1.52 & 0.74 & 1.43 & 3.12 \\
\hline & $45 / 15$ & 4.09 & 2.73 & 3.83 & 1.54 & 1.14 & 2.66 \\
\hline \multirow{2}{*}{ UK } & $30 / 30$ & 2.77 & 1.62 & 1.52 & 0.74 & 1.43 & 1.24 \\
\hline & $45 / 15$ & 2.97 & 1.46 & 1.59 & 0.66 & 0.92 & 1.26 \\
\hline \multirow[b]{2}{*}{ RSM } & $30 / 30$ & 7.71 & 7.41 & 8.48 & 5.46 & 6.60 & 8.42 \\
\hline & $45 / 15$ & 63.69 & 26.49 & 25.12 & 29.83 & 47.03 & 11.24 \\
\hline \multirow[t]{2}{*}{ Square Cup } & & \multicolumn{3}{|c|}{ Maximum Equivalent Plastic Strain } & \multicolumn{3}{|c|}{ "Maximum Thinning } \\
\hline & Train/Test & DC06 & DP600 & HSLA340 & DC06 & DP600 & HSLA340 \\
\hline \multirow{2}{*}{ SK } & $30 / 30$ & 0.31 & 0.42 & 0.71 & 1.92 & 5.98 & 4.44 \\
\hline & $45 / 15$ & 0.30 & 0.40 & 0.72 & 2.19 & 4.05 & 4.07 \\
\hline \multirow{2}{*}{ OK } & $30 / 30$ & 0.32 & 0.42 & 0.71 & 1.92 & 5.98 & 4.45 \\
\hline & $45 / 15$ & 0.30 & 0.40 & 0.71 & 2.19 & 4.08 & 4.09 \\
\hline \multirow[b]{2}{*}{ UK } & $30 / 30$ & 0.23 & 0.42 & 0.70 & 1.92 & 6.66 & 3.46 \\
\hline & $45 / 15$ & 0.30 & 0.40 & 0.71 & 2.19 & 2.89 & 3.93 \\
\hline \multirow{2}{*}{ RSM } & $30 / 30$ & 7.51 & 7.46 & 9.92 & 6.81 & 13.13 & 9.48 \\
\hline & $45 / 15$ & 25.95 & 35.6 & 3.38 & 337.89 & 406.71 & 18.14 \\
\hline
\end{tabular}

The RSM metamodels generated with $n=30$ training simulations and evaluated with $k=30$ testing simulations achieve values of RMSRE ranging from 5.46\% in case of maximum thinning in the U-Channel process using DC06 (see Table 2), to $13.13 \%$ in case of maximum thinning in the Square Cup deep-drawing using DP600. Globally, the RSM metamodels generated with 45 training simulations present higher RMSRE values than those obtained with 30 training simulations (the only exception being the prediction of maximum equivalent plastic strain in the Square Cup deep-drawing using HSLA340). This can be associated with metamodel overfitting (forced fitting) to the training points, leading to poor prediction capabilities (i.e. high values of RMSRE). Figure 2(a) shows the correspondence between the numerical simulation and predicted responses of maximum thinning in the U-Channel, with DC06, using Universal Kriging and RSM metamodels with 30 ("RSM (30/30)") and 45 ("RSM (45/15)") training simulations. The dispersion in the RSM results, namely RSM (45/15), is more pronounced than in Kriging; the case of RSM with 30 training simulations generates a more balanced metamodel than RSM (45/15). Figure 2(b) shows the evolutions of $\|\boldsymbol{\beta}\|$ and RMSRE with the number of training points (from 30 to 60). The idea is to analyse the separated influence of the constant, linear, interaction and quadratic terms (see Equation 1) in the values of RMSRE, through the respective 
values of $\beta_{0},\left\|\beta_{\mathrm{i}}\right\|,\left\|\beta_{\mathrm{ij}}\right\|$ and $\left\|\beta_{\mathrm{ii}}\right\|$. According to this figure, similar evolutions with the number of training points of RMSRE and $\left\|\beta_{\mathrm{ij}}\right\|$ (related with the interaction terms) are observed. When the number of training points is equal to 45 , the RSM metamodel overfits the training points, leading to a peak value of RMSRE associated to a relatively high value of $\left\|\beta_{\mathrm{ij}}\right\|$, when compared to the values of $\left\|\beta_{\mathrm{i}}\right\|$ and $\left\|\beta_{\mathrm{ii}}\right\|$. When the number of training points is less or higher than 45 , the relative values of $\left\|\beta_{\mathrm{i}}\right\|,\left\|\beta_{\mathrm{ij}}\right\|,\left\|\beta_{\mathrm{ii}}\right\|$ and RMSRE reduce. In the first case ( $\left.<45\right)$, the least squares solution leads to interpolation to the training points with relatively low values of $\left\|\beta_{\mathrm{i}}\right\|,\left\|\beta_{\mathrm{ij}}\right\|$ and $\left\|\beta_{\mathrm{ii}}\right\|$, close to each other. In the latter case (>45), interpolation does not occur and the least squares solution leads to relatively low values of $\left\|\beta_{i}\right\|,\left\|\beta_{i j}\right\|$ and $\left\|\beta_{\mathrm{ii}}\right\|$, close to zero. In both cases, near-constant RSM metamodels are generated, i.e. the values of $\beta_{0}$ and $\|\boldsymbol{\beta}\|$ are relatively close to each other.

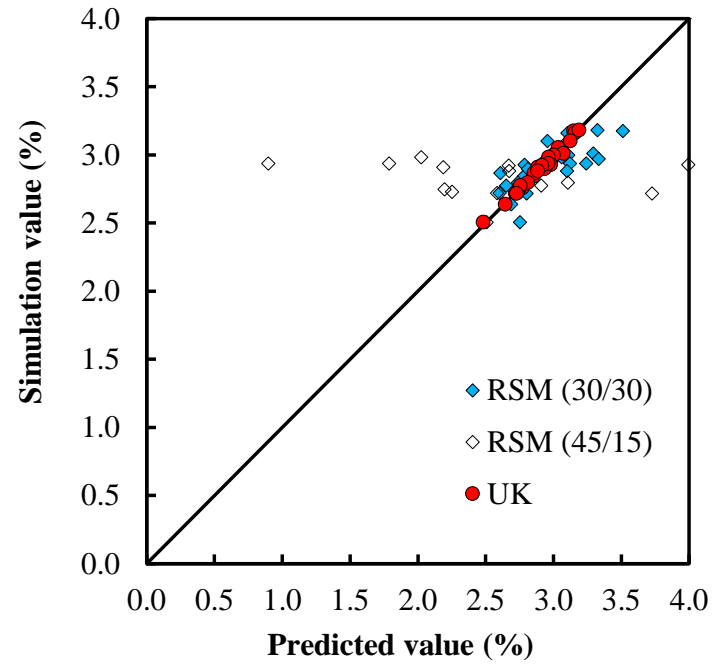

(a)

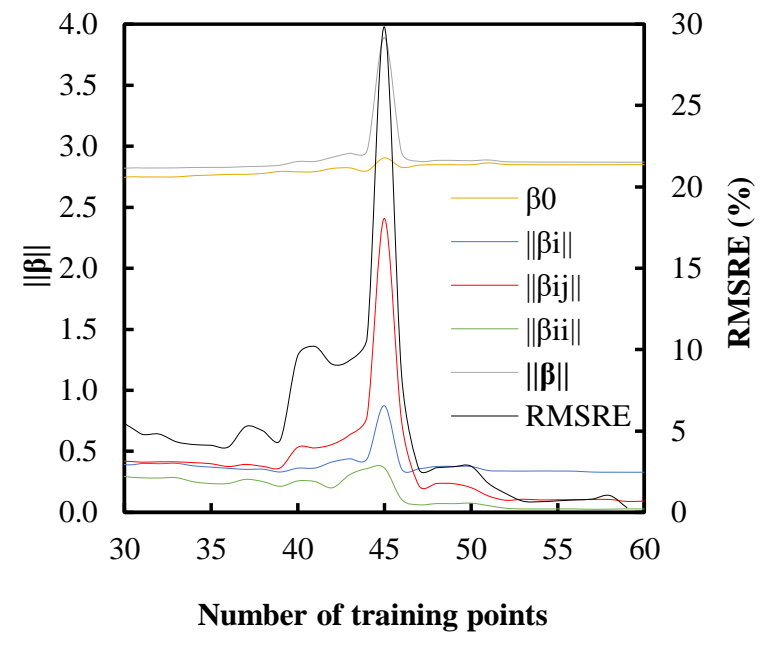

(b)

FIGURE 2. (a) Correspondence between the simulated and predicted response values of maximum thinning in the U-Channel process with DC06, obtained by UK and RSM metamodels; (b) $\|\boldsymbol{\beta}\|$ and RMSRE vs. number of RSM training points.

\section{CONCLUSIONS}

In this work, Kriging and RSM metamodeling techniques were applied to describe the variability in the results of two sheet metal forming processes, the U-Channel and Square Cup processes. In general, Kriging (namely Universal Kriging) proved to be the most reliable metamodeling technique, allowing accurate response predictions for all study cases. The performance of the RSM metamodels showed to be strongly dependent on the number of design (training) points, which was not the case for Kriging metamodels. In this context, it can be concluded that the performance of the metamodels depends not only on the type of response, material and forming process under analysis, but it may also strongly depend on the total number of training points used to generate the metamodels.

\section{ACKNOWLEDGMENTS}

This work was supported by the projects RDFORMING and EZ-SHEET, co-funded by Portuguese Foundation for Science and Technology (FCT), by FEDER, through the program Portugal-2020 (PT2020), and by POCI (refs. POCI01-0145-FEDER-031243 and POCI-01-0145-FEDER-031216). All supports are gratefully acknowledged.

\section{REFERENCES}

1. P. A. Prates, A. S. Adaixo, M. C. Oliveira, J. V. Fernandes, Int. J. Adv. Manuf. Technol. 96, 561-580 (2018).

2. C. Huang, B. Radi, A. El Hami, Int. J. Adv. Manuf. Technol. 86, 3229-3240 (2016).

3. L. Marretta, R. Di Lorenzo, Int. J. Adv. Manuf. Technol. 51, 117-134 (2010).

4. L.F. Menezes, C. Teodosiu, J. Mater. Process. Technol. 97, 100-106 (2000).

5. D. M. Neto, M. C. Oliveira, L. F. Menezes, Arch. Comput. Methods Eng. 24, $37-87$ (2017). 